haemoglobin prior to endoscopy was $92 \mathrm{~g} / \mathrm{L}$. $40 \%$ were on anticoagulants or anti-platelets on admission. Of all patients who needed Hemospray, 43 had gastroscopies, 1 ERCP and 1 had colonoscopy. The details of diagnosis are in table 1 .

- $23.39(86 \%)$ patients had Hemospray as adjunct therapy \& all patients had complete haemostasis documented at the end of index procedure.

- 24. It was used as the only modality of therapy successfully in 6 patients (4 with malignancy and 2 iatrogenic bleeds)

- 25. Overall, 6/45 patients had a documented rebleed after discharge but within 4 weeks; 4 had bleeding vessels and were on warfarin or Ticagrelor; 2 patients had tumour related recurrent bleeding.

- 26. Forrest classification applicable to 22 patients $-1 \mathrm{~A}(\mathrm{n}=3$, $15 \%), 1 \mathrm{~B}(\mathrm{n}=10,46 \%), 2 \mathrm{~A}(\mathrm{n}=3,15 \%), 2 \mathrm{~B}(\mathrm{n}=3,15 \%), 2 \mathrm{C}$ $(\mathrm{n}=2,9 \%)$.

- $27.100 \%(n=7)$ of UGIB related to malignancy responded to Hemospray (adjunct and monotherapy included)

- 28. 30 day mortality was $24 \%(n=11)$ with 3 deaths directly attributed to GI bleeding (4 palliative secondary to malignancy and 4 multifactorial in elderly patients).

Conclusions Our 4 year experience suggests that Hemospray can be a useful adjunct to conventional endoscopic therapy for acute GI bleed with success rates comparable to previous reports (nearly $85 \%$ in our cohort), with a rebleed rate of $14 \%$. Our findings suggest it could be useful as monotherapy for bleeding related to malignancies where other therapy may be impractical. More studies with larger numbers are needed to add to the evidence base.

\begin{tabular}{ll} 
Abstract PTH-056 Table 1 & \\
\hline Diagnosis & Number $\mathbf{n}=45$ \\
\hline Duodenal Ulcer & $14(32 \%)$ \\
Oesophageal/gastric ulcer & $4(9 \%)$ \\
Malignancy related & $7(15 \%)$ \\
latrogenic bleeding & $7(15 \%)$ \\
Other including unclear cause of bleed & $13(29)$ \\
\hline
\end{tabular}

\section{PTH-057 ENDOSCOPIC MANAGEMENT OF BURIED BUMPER SYNDROME}

Angad Singh*, Andrea Cartwright, Rizwan Kassam, Javaid Subhani. Basildon University Hospital, Basildon, UK

\subsection{6/gutjnl-2018-BSGAbstracts.78}

Introduction Buried Bumper Syndrome (BBS) is an uncommon, yet potentially serious complication of percutaneous gastrostomy tube (PEG) placement with a quoted incidence of $1 \%$. Usually identified during routine nursing assessment, release of a buried bumper can be achieved via external manipulation, endoscopically or via surgery.

Aim Review the frequency and success of endoscopic treatment of BBS.

Methods At Basildon University Hospital a 4-level stepwise approach was adopted for management of Buried Bumper Syndrome, with each level representing an escalation of therapy.
- 29. Level 1- Release achieved by external manipulations by a nurse or radiologist.

- 30. Level 2- Endoscopic if the internal bumper can be seen through a relatively wide tract it may be pulled through by graspers or snare.

- 31. Level 3-Sometime the tract is narrow and may require quadrantic incisions ( $3 a$ ) or subsequent balloon dilations ( $3 b$ ) before release.

- 32. Level 4- Cannot be endoscopically released and require surgery.

Retrospective review of endoscopy records held on Unisoft from August 2009 to Jan 2018 by a bespoke Access query. Endoscopy and clinical records were reviewed of the identified patients. By definition no Level 1 cases would be identified.

Results 27 incidences of BBS requiring 30 endoscopic procedures in 18 patients were identified. 4 (22\%) patients had repeated distinct episodes of BBS (2-5). 18 episodes of BBS were successful resolved endoscopically (67\%). Interventions were 4x Level 2, 9x level 3a, 5x Level 3b.

Endoscopic treatment of 9 episodes of BBS were unsuccessful (Level 4). 4/9 patients had a surgical removal, though one precipitated a 2 month admission complicated by an ITU stay. The other 5 patients were deemed unfit, though one patient continued to suffer from septic episodes related to the bumper.

The PEG insertion date was unknown many patients (e.g out of area, pre-2009) but for 12 patients the time from insertion to attempted release of BBS was known. 2 episodes $<12$ months, 3 occurred at 12 months, 3 at $21-25$ months, 4 at $31-33$ months. The only short (2 months) interval was in a patient with previous PEG.

Conclusion This is a relatively infrequent endoscopic dilemma with only 27 instances over a 8.5 year study period. However with rare scenarios the referral pathway can be unclear. Only one endoscopist managed Level 3 releases and by default they became the routine destination for Nutrition team referrals. This allowed a $66 \%$ success rate but disappointingly $22 \%$ of the patients had recurrent episodes. Recommendations 1) Education of the institutions caring for these patients. 2) Single endoscopists to develop expertise in each centre 3) If successful, attempt to site a new PEG in a second area despite the difficulty.

\section{PTH-058 SMART COLONOSCOPY:USING BIG-DATA TO IDENTIFY PREDICTORS OF NORMAL COLONOSCOPIC EXAMINATIONS}

Matt Stammers, Sreedhari Thalasekaran, Pradeep Bhandari. Queen Alexandra Hospital, Portsmouth, UK

\subsection{6/gutjnl-2018-BSGAbstracts.79}

Introduction Endoscopy workload is increasing at a faster pace than available resources. The NHS has a wealth of data, which if used properly can improve resource allocation in future.

The aim of this study was to review mass colonoscopy data to identify those factors most associated with a normal examination, in order to help rationalise future resource utilisation.

Methods We constructed a standardised, anonymised database, containing all colonoscopies performed locally between 01/01/ 
2010 and 10/12/2016. The records were then histology matched.

The data was then analysed using the Anacondas ${ }^{\mathrm{TM}} 3$ distribution of Python, using numpy, pandas, matplotlib and seaborn to clean and prepare, plot and perform statistical analysis on the data.

Results 23837 colonoscopies were performed on 18489 individual adults during the study period.

544 procedures had to be excluded as they lacked an NHS number and couldn't be histology matched. 23293 procedures remained.

$50.4 \%$ of the procedures were performed on females. The median age was 64. Across all the procedures, 25.46\% were reported as entirely normal by the endoscopist. $3.04 \%$ of procedures contained a histologically confirmed cancer.

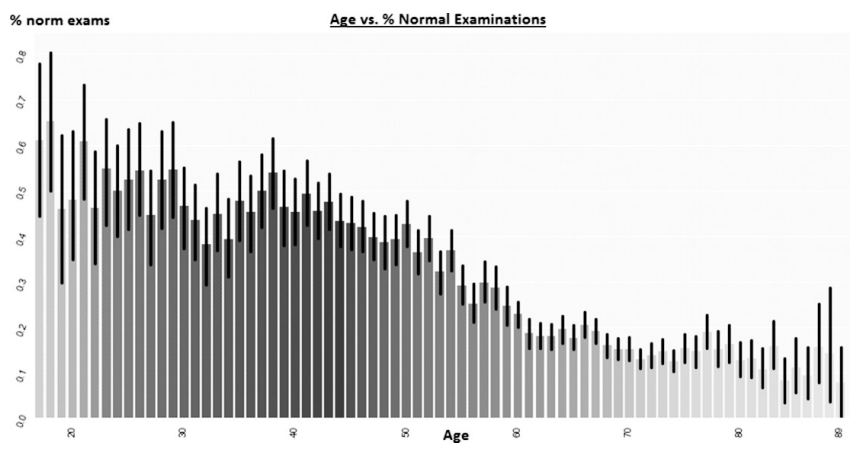

Abstract PTH-058 Figure 1 Age vs\% normal examinations

Age We found that the chances of obtaining a normal examination declined from $\sim 49 \% \pm 5 \% \leq 43$ years to $\leq 20 \% \pm 2 \%$ in those $\geq 61$ years.

In patients aged $\leq 43$ OR of a normal exam=3.29. For those aged $\geq 61$, (OR $=0.32$ for a normal exam). Note, all OR's in this study had $\mathrm{p}<0.0001$ significance.

Sex Examinations performed on females were more likely to be reported as normal compared to men $(\mathrm{OR}=1.73)$. For women $\leq 43$, OR for normality $=3.88$.

Priority Routine priority was strongly associated with normal colonoscopy, $(\mathrm{OR}=1.99)$. Routine procedures on females $\leq 43$ were very likely to be normal $(\mathrm{OR}=4.90)$. These patients were very unlikely to have cancer $(\mathrm{OR}=\mathbf{0 . 0 9 3})$.

Indications Abdominal pain, anaemia (iron deficient) and bowel habit changes (of all types) and family history of colonic cancer were all found to be associated with $\mathrm{a} \geq 40 \%$ rate of normal examinations, $(\mathrm{OR}=3.57)$.

The highest incidence of normal examinations was found amongst women $\leq 43$ undergoing routine colonoscopy for abdominal pain $(\mathrm{OR}=7.85)$, followed by bowel habit changes $(\mathrm{OR}=6.49)$, and anaemia $(\mathrm{OR}=5.91)$.

Conversely, the highest rates of pathology were found in men $\geq 61$ undergoing bowel cancer screening, (OR for pathology $=4.98$; OR for malignancy $=2$ ).

Conclusions We have developed a method for performing mass data analysis to identify trends in endoscopy data.

Our data can help improve future utilisation of other colonic investigational modalities like colon capsule for low risk individuals. This can release colonoscopy capacity for the patients most at need.

\section{PTH-059 ANALYSIS OF 140000 PATIENTS WITH BILE DUCT STONES PRESENTING TO ENGLISH ACUTE TRUSTS, 2013-2016}

${ }^{1}$ Maxime Delvincourt ${ }^{*},{ }^{2}$ Jodi Carter, ${ }^{2}$ Keith Bodger, ${ }^{3}$ George Webster, ${ }^{1}$ Richard Sturgess. ${ }^{1}$ Aintree University Hospital NHS Trust, Liverpool, UK; ${ }^{2}$ Methods Analytics, London, UK; ${ }^{3}$ University College London Hospital Trust, London, UK

10.1136/gutjnl-2018-BSGAbstracts.80

Common ile uct (CBD) stones 10\%-20\% of patients with gallstone disease. ndoscopic retrograde cholangio-pancreatography (ERCP)

Methods All adult patients with diagnosis (ICD-10) codes for alculus of bile duct with cholangitisalculus of bile duct with cholecystitisalculusofbile duct without cholangitis were used to select results from HospitalEpisode Statistics (HES) data for the years 2013/14 - 2016/17. Episodes were excluded where there were episodes with one or moreof these codes for the previous 2 financial years to censor patients already on a CBD pathway.

Results There were 140890 patients identified, of which $91178(64.72 \%)$ were female Mean age at first admission was 70. Of the 140890 individual patients, $102072(72 \%)$ had an emergency first admission. The incidence of cases in each year was 33,557, 35,053, 35621 and 36659 respectively. were performed on 48306 patients in this cohort over the study period of the 48306 individuals who had an ERCP, 39192 (65\%) had only one procedure, 7105 (1\%) had two procedures and $2009(\%)$ had three or more.

For the identified CBD cohort 60793 holecystectomies were performed over the study period with 17063 patients having had both an ERCP and holecystectomy.

\section{PTH-060 AN ASSESSMENT OF QUALITY OF, AND FACTORS AFFECTING, OESOPHAGOGASTRODUODENOSCOPY (OGD)}

David Tai ${ }^{\star}$, Gloria SZ Tun, Mustafa Jalal, Stefania Chetcuti Zammit, Mark McAlindon. Sheffield Teaching Hospitals NHS Trust, Sheffield, UK

\subsection{6/gutjnl-2018-BSGAbstracts.81}

Introduction Oesophagogastroduodenoscopy (OGD) is the accepted gold standard investigation for upper GI symptoms although quality has rarely been assessed. In this study, quality of OGD and the effects of sedation, procedural tolerance and duration of examination were investigated.

Methods Consecutive out-patients undergoing OGD were recruited. Patients scored (none-severe: 0-10) procedural distress, abdominal discomfort (AD) and pain (AP). Video recordings of the OGDs were reviewed, scoring the views (score 1$5:<25 \%, 25 \%-50 \%, 50 \%-75 \%,>75 \%$ and $100 \%$ of mucosa seen) of eight upper GI stations and time spent in retroflexion and in the duodenum. Scores and times are reported as mean ( \pm SEM).

Results OGD was performed on forty patients $(58 \pm 3$ years old, $62.5 \%$ male, $25 \%$ sedated) by one of three endoscopists. There was a significant difference between visualisation scores (oesophagus, $4.5 \quad( \pm 0.1)$; gastro-oesophageal junction, 4.5 $( \pm 0.1)$; cardia, $4.0( \pm 0.2)$; fundus, $3.7( \pm 0.2)$; upper body, $3.8( \pm 0.1)$; distal body, $4.1( \pm 0.1)$; incisura, $4.0( \pm 0.2)$ and antrum, $4.9( \pm 0.1)$; Kruskal-Wallis $\mathrm{H} \quad$ Test: $\mathrm{H}(7)=60.2$; 